\title{
Knockdown of CHCHD2 inhibits migration and angiogenesis of human renal cell carcinoma: A potential molecular marker for treatment of $\mathbf{R C C}$
}

\author{
QIAN $\mathrm{CHENG}^{1 *}$, DEBAO QU ${ }^{1,2^{*}}, \mathrm{ZHENG} \mathrm{LU}^{1}$ and LONGZHEN ZHANG ${ }^{2,3}$ \\ ${ }^{1}$ Cancer Institute; ${ }^{2}$ Department of Radiation Oncology, The Affiliated Hospital of Xuzhou Medical University; \\ ${ }^{3}$ Jiangsu Center for The Collaboration and Innovation of Cancer Biotherapy, Cancer Institute, \\ Xuzhou Medical University, Xuzhou, Jiangsu 221002, P.R. China
}

Received February 20, 2017; Accepted June 5, 2018

DOI: $10.3892 / 01.2018 .9686$

\begin{abstract}
Coiled-coil-helix-coiled-coil-helix domaincontaining protein 2 (CHCHD2), a novel cell migration determinant, is able to co-express with other genes of the oxidative phosphorylation pathway by using a computational expression screening technique. However, little is known about the expression and biological function of CHCHD2 in human renal cell carcinoma (RCC). Western blotting was performed to detect CHCHD2 expression levels in normal renal cells and carcinoma cells. Immunohistochemistry was performed to detect an association between CHCHD2 expression and clinicopathological parameters in $75 \mathrm{RCC}$ tissues using a tissue microarray. The function of CHCHD2 in the migration and angiogenesis of RCC cells was investigated using Transwell migration and tube formation assays. $\mathrm{CHCHD} 2$ expression was markedly increased in human RCC cells. The results of immunohistochemical analysis revealed that CHCHD2 expression was markedly associated with tumor grade $(\mathrm{P}<0.001)$. Notably, CHCHD2 knockdown inhibited RCC migration and tube formation of human umbilical vascular endothelial cells. CHCHD2 knockdown further suppressed matrix metalloproteinase-2 protein levels and enzyme activity. An ELISA identified that CHCHD2 knockdown decreased the secretion of vascular endothelial growth factor. The gathered data disclose information on the association of CHCHD2 with migration and angiogenesis of human RCC, and may strengthen the feasibility of targeting CHCHD2 as a potential therapeutic target.
\end{abstract}

Correspondence to: Dr Longzhen Zhang, Department of Radiation Oncology, The Affiliated Hospital of Xuzhou Medical University, 99 West Huai Hai Road, Xuzhou, Jiangsu 221002, P.R. China E-mail: jsxzzlz@126.com

*Contributed equally

Key words: coiled-coil-helix-coiled-coil-helix domain-containing protein 2, human renal cell carcinoma, migration, angiogenesis

\section{Introduction}

Renal cell carcinoma (RCC) accounts for $90 \%$ of renal cancer cases, and is the most common carcinoma of the adult kidney (1). The incident rate of RCC has risen during the previous decade (2), making it the 7 and 8 th most common cancer in the USA among males and females, respectively (3). Although surgery is currently the most effective treatment approach for localized RCC tumors and commonly used molecular drugs have improved over the past several decades (4), $30 \%$ of patients continue to develop metastatic disease following surgical resection (5). Development of specific targets in relevant biological pathways has been the primary advancement in treatment (6). In spite of these advances RCC continues to be a devastating cancer with a low number of effective treatment options. Therefore, increased efforts should be made to improve early diagnosis and the monitoring of patients, particularly patients that undergo resection and treatment for metastatic RCC.

Coiled-coil-helix-coiled-coil-helix domain-containing protein 2 (CHCHD2), a member of a family of proteins containing the $\mathrm{CHCH}$ domain, was identified to be co-expressed with other genes of the oxidative phosphorylation pathway by using a computational expression screening technique (7). In addition, Seo et al (8) revealed that CHCHD2 may be identified by an in vitro functional genetic selection strategy as a novel cell migration determinant. Liu et al (9) reported that CHCHD2 is able to inhibit apoptosis by interacting with $\mathrm{B}$ cell lymphoma-extra large (Bcl-xL) to regulate Bcl-associated X protein (BAX) activation. However, less is known regarding the expression and biological function of CHCHD2 in RCC.

In the present study, CHCHD2 staining in RCC cells lines and tissues was evaluated. Then, the association between CHCHD2 expression and clinicopathological variables was assessed. The possible association between CHCHD2, and movement and angiogenesis of RCC was also investigated. It was identified that $\mathrm{CHCHD} 2$ expression was increased in human RCC cells and tissue. CHCHD2 expression was significantly increased in tumor grades II-IV compared with grade I in accordance with the World Health Organization 
classification $(\mathrm{P}<0.001)(10,11)$; however, no significant association was identified between CHCHD2 expression and other clinicopathological variables, including age, tumor size and Tumor-Node-Metastasis (TNM) stage $(10,11)$. The present study identified that the migratory capacity of RCC was inhibited following suppression of CHCHD2 expression using a stable small interfering (si)RNA compared with a scrambled siRNA control in vitro. The matrix metalloproteinase-2 (MMP-2) protein level and enzyme activity were inhibited following CHCHD2 knockdown, confirmed using western blotting and gelatin zymography. In addition, data revealed that tube formation of human umbilical vascular endothelial cells (HUVECs) was suppressed following CHCHD2 knockdown, and the ELISA assay identified that the secretion of vascular endothelial growth factor was inhibited following CHCHD2 knockdown. Taken together these data indicate that CHCHD2 may be a novel target for the treatment of RCC.

\section{Materials and methods}

Ethics statement. The Institutional Review Board of Xuzhou Medical University (Xuzhou, China) approved the present study, conducted in accordance with the approved guidelines. All patients provided written informed consent for their colorectal tissue samples to be used in the present study.

Patients and samples. The suprarenal epithelioma tissue microarray (TMA) was purchased from Shanghai Outdo Biotech (Shanghai, China) (http://superchip.bioon.com.cn/). It included 75 patients (mean age, 59) who underwent radical nephrectomy between 2006 and 2008. In this TMA, the array dot diameter was $1.5 \mathrm{~mm}$ and each dot represented a tissue spot from one individual specimen. The patients consisted of $50(66.7 \%)$ men and $25(33.3 \%)$ women. A total of $42(56 \%)$ patients were aged $\leq 57$ years and $32(42.7 \%)$ ages $>57$ years. A total of 57 (76\%) patients were classified TNM I-II and 18 (24\%) TNM stage III-IV. Details of the patient characteristics and CHCHD2 expression are provided in Table I. Additionally, three RCC tissues and paired non-cancerous tissues were obtained from The Affiliated Hospital of Xuzhou Medical University. Patients with ccRCC who underwent radical nephrectomy without prior treatment were recruited from the Affiliated Hospital of Xuzhou Medical University between January 2006 and December 2008. The clinicopathological information, including age at diagnosis, sex, tumor diameter, depth of invasion, lymph node metastasis and TNM stage, was obtained from the medical records in the Affiliated Hospital of Xuzhou Medical University. All the tissue specimens were obtained for the present research with patients' informed consent, and the use of human specimens was approved by the Review Board of the Affiliated Hospital of Xuzhou Medical University.

Cell lines and transfection. All cell lines were obtained from the Shanghai Institute of Biochemistry and Cell Biology, Chinese Academy of Sciences (Shanghai, China). HK-2 and ACHN were grown in Dulbecco's modified Eagle's medium (DMEM; Invitrogen; Thermo Fisher Scientific, Inc., Waltham, MA, USA) supplemented with $15 \%$ fetal bovine serum (FBS; Invitrogen; Thermo Fisher Scientific, Inc.), while 786-O and Ketr-3 cells were cultured in RPMI-1640 (Invitrogen; Thermo
Fisher Scientific, Inc.) supplemented with $10 \%$ FBS. All cells were maintained as monolayer cultures at $37^{\circ} \mathrm{C}$ in a humidified incubator with an atmosphere of $5 \% \mathrm{CO}_{2}$.

The CHCHD2 siRNA and scrambled siRNA were purchased from GenePharma (Shanghai, China) (Stealth RNAi targeting CHCHD2 5'-GGGCACACATTGGGTCACGCC ATTA-3'). Transfection of $1.25 \mu 120 \mathrm{mM}$ siRNAs into $40 \%$ confluent 786-O and ACHN cells was performed using siLentFect Lipid reagent (Bio-Rad Laboratories, Inc., Hercules, CA, USA) according to the manufacturer's protocol. Cell migration assays were performed $48 \mathrm{~h}$ after transfection.

Immunohistochemistry of TMA. Immunohistochemistry was performed using a standard avidin biotinylated-HRP complex (ABC) kit (Zhongshan Belling Biotechnology Co., Ltd., Beijing, China). TMA sections were deparaffinized in xylene and then rehydrated with graded ethanol and distilled water. According to the manufacturer's protocol, diaminobenzidine (DAB; Zhongshan Jinqiao Biotechnology Co., Ltd., Beijing, China) was used to produce a brown precipitate. TMA slides were incubated with homemade rabbit anti-CHCHD2 antibody (1:200 dilution; a gift from Dr Yanping Zhang, University of North Carolina at Chapel Hill, Chapel Hill, NC, USA) overnight at $4^{\circ} \mathrm{C}$, and incubated with a goat anti-rabbit biotinylated secondary antibody (ready to use; catalog no. PV-9001, Jinqiao Biotechnology Co., Ltd.) at room temperature for $30 \mathrm{~min}$. The sections were incubated with streptavidin-peroxidase (Zhongshan Belling Biotechnology Co., Ltd.) at room temperature for an additional $30 \mathrm{~min}$, washed with phosphate-buffered saline (PBS) and stained using DAB at room temperature for $15 \mathrm{~min}$. Subsequently, sections were rinsed in distilled water and counterstained with hematoxylin (Beyotime Institute of Biotechnology, Shanghai, China) at room temperature for $30 \mathrm{sec}$. Using a light microscope (Olympus BX-51 light microscope) and a Camedia Master C-3040 digital camera (both from Olympus Corporation, Tokyo, Japan), the CHCHD2 expression in TMA was identified as positive when $\geq 5 \%$ of tumor cells exhibited immunopositivity, while biopsies with $<5 \%$ tumor cells exhibiting immunostaining were graded negative. Samples with an immunoreactivity score (IRS) of 0-3 and 4-12 were classified as weak and strong expression of CHCHD2, respectively.

Cell migration assays. The cell migration assay was executed using $6.5 \mathrm{~mm}$ Transwell Boyden chambers (pore size, $8 \mu \mathrm{m}$; Corning Incoporated, Corning, NY, USA) as described previously, but with a number of modifications (12). Cell suspension $(100 \mu \mathrm{l})$ without serum at a density of $4 \times 10^{4}$ cells/well was seeded in the Transwell chambers. After $12 \mathrm{~h}$ for migration assays, cells in the upper chamber were carefully removed with a cotton swab. The cells that had migrated to the basal side of the membrane were fixed in $100 \%$ methanol at room temperature for $30 \mathrm{~min}$ and stained with crystal violet $(0.04 \%$ in water, $100 \mu \mathrm{l}$; Beyotime Institute of Biotechnology) at room temperature for 15-30 min. The permeating cells were counted under an inverted microscope (Ti-U; Nikon Corporation, Tokyo, Japan) and images were captured at a magnification of x10.

Western blotting and antibodies. Cells were transiently transfected with siRNA for $48 \mathrm{~h}$. The cells were then lysed with $0.5 \%$ NP-40 lysis buffer and protein concentrations 
Table I. CHCHD2 staining and clinicopathological characteristics of 75 patients with renal cancer.

\begin{tabular}{|c|c|c|c|c|}
\hline \multirow[b]{2}{*}{ Variables } & \multicolumn{4}{|c|}{ CHCHD2 staining } \\
\hline & Weak, n (\%) & Strong, n (\%) & Total, $\mathrm{n}$ & P-value ${ }^{a}$ \\
\hline Age, years & $38(50.7)$ & $37(49.3)$ & 75 & \\
\hline$\leq 56$ & $19(52.8)$ & $17(47.2)$ & 36 & 0.725 \\
\hline$>56$ & $19(48.7)$ & $20(51.3)$ & 39 & \\
\hline \multicolumn{5}{|l|}{ Sex } \\
\hline Male & $25(50.0)$ & $25(50.0)$ & 50 & 0.870 \\
\hline Female & $13(52.0)$ & $12(48.0)$ & 25 & \\
\hline \multicolumn{5}{|c|}{ Tumor size, $\mathrm{cm}$} \\
\hline$\leq 7$ & $24(55.8)$ & $19(44.2)$ & 43 & 0.301 \\
\hline$>7$ & $14(43.8)$ & $18(56.2)$ & 32 & \\
\hline \multicolumn{5}{|l|}{ Grade } \\
\hline I & $2(66.7)$ & $1(33.3)$ & 3 & $<0.001$ \\
\hline II & $29(80.6)$ & 7 (19.4) & 36 & \\
\hline III & $7(25.0)$ & $21(75.0)$ & 28 & \\
\hline IV & $0(0.0)$ & $8(100.0)$ & 8 & \\
\hline \multicolumn{5}{|l|}{ TNM stage } \\
\hline I & $21(63.6)$ & $12(36.4)$ & 33 & 0.166 \\
\hline II & $11(45.8)$ & $13(54.2)$ & 24 & \\
\hline III & $6(35.3)$ & $11(64.7)$ & 17 & \\
\hline IV & $0(0.00)$ & $1(100.0)$ & 1 & \\
\hline
\end{tabular}

${ }^{a} \chi^{2}$ test. CHCHD2, coiled-coil-helix-coiled-coil-helix domain-containing protein 2; TNM, Tumor-Node-Metastasis.

were assessed using a bicinchoninic acid assay. The loading protein $(100 \mu \mathrm{g})$ was separated by $12 \%$ SDS-PAGE under reducing conditions, and then transferred onto nitrocellulose membranes. The membranes were blocked at room temperature for $2 \mathrm{~h}$ in Blotto (5\% skimmed milk and PBS). The primary antibodies utilized at $4^{\circ} \mathrm{C}$ overnight were rabbit anti-CHCHD2 (1:1,000; supplied by Dr Yanping Zhang), rabbit anti-MMP2 (1:1,000; catalog no. 4022s; Cell Signaling Technology, Inc., Danvers, MA, USA) and mouse anti- $\beta$-actin (1:5,000; catalog no. BM0626; Wuhan Boster Biological Technology, Wuhan, China). Each antibody was used according to the manufacturer's protocol. Fluorescence-conjugated secondary antibody (IRDye 700/800; Rockland Immunochemicals Inc., Limerick, PA) was used at a dilution of 1:10,000 at room temperature for $2 \mathrm{~h}$. Analysis was performed using the Odyssey Infrared Imaging system (LI-COR Biosciences, Lincoln, NE, USA). All experiments were performed at least three times unless otherwise indicated.

Gelatin zymography analysis. The gelatinolytic activity was analyzed using gelatin zymography according to the method previously described with a number of modifications (13). Prior to gelatin zymography, cells were cultured in serum-free conditioned culture medium overnight. Next, the culture conditioned medium was harvested and the proteins in the medium were concentrated with Amicon Ultra-4-30 k centrifugal filters (EMD Millipore, Billerica, MA, USA) at $7,500 \mathrm{x} g$ for $20 \mathrm{~min}$ at $4^{\circ} \mathrm{C}$. A total of $30 \mu \mathrm{g}$ protein was loaded in non-redenaturing conditions on a $10 \%$ polyacrylamide gel containing $0.1 \%$ gelatin. The SDS was removed from the gel using $2.5 \%$ Triton $\mathrm{X}-100$ for $1 \mathrm{~h}$ at room temperature. The gel was incubated overnight at $37^{\circ} \mathrm{C}$ in development buffer (20 mM Tris-HCl, pH 8.0, $150 \mathrm{mM} \mathrm{NaCl}, 5 \mathrm{mM} \mathrm{CaCl}_{2}$, and $0.01 \% \mathrm{NaN}_{3}$ ), stained with $0.5 \%$ Coomassie blue R250 (Sigma-Aldrich; Merck KGaA, Darmstadt, Germany) at room temperature for $1 \mathrm{~h}$, and destained with $30 \%$ methanol and $10 \%$ glacial acetic acid for $2 \mathrm{~h}$ at room temperature.

Tube formation assay. Transfected 786-O and ACHN cells $\left(1 \times 10^{6}\right)$ were cultured in a 6 -well plate with fresh complete medium for $48 \mathrm{~h}$, then collected and centrifuged at $800 \mathrm{x} \mathrm{g}$ at room temperature for $5 \mathrm{~min}$ to remove any cell debris prior to its use as a conditioned medium. Next, 48-well plates coated with Matrigel were kept at $37^{\circ} \mathrm{C}$ for 30 min prior to the endothelial cell tube formation assay. HUVECs (Shanghai Institute of Biochemistry and Cell Biology, Chinese Academy of Sciences, Shanghai, China) $\left(2 \times 10^{4} /\right.$ well) were suspended in $100 \mu 1$ conditioned medium and applied to the pre-coated 48-well plate. The number of capillary-like tubes was quantified after $24 \mathrm{~h}$ in three random microscopic fields (magnification, $\mathrm{x} 10$ ) with a computer-assisted microscope (Nikon Corporation).

ELISA for VEGF. In order to determine the concentration of VEGF, 786-O and ACHN cells were transfected with siRNA subsequent to being plated in 6-well tissue culture plates at a density of $1 \times 10^{6}$ cells/well. The supernatants were harvested 
A
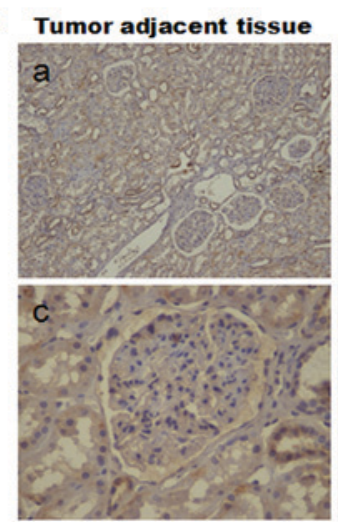

C

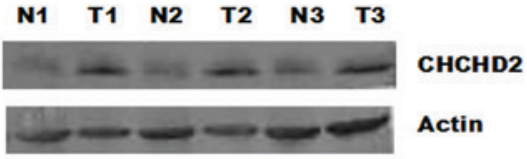

B
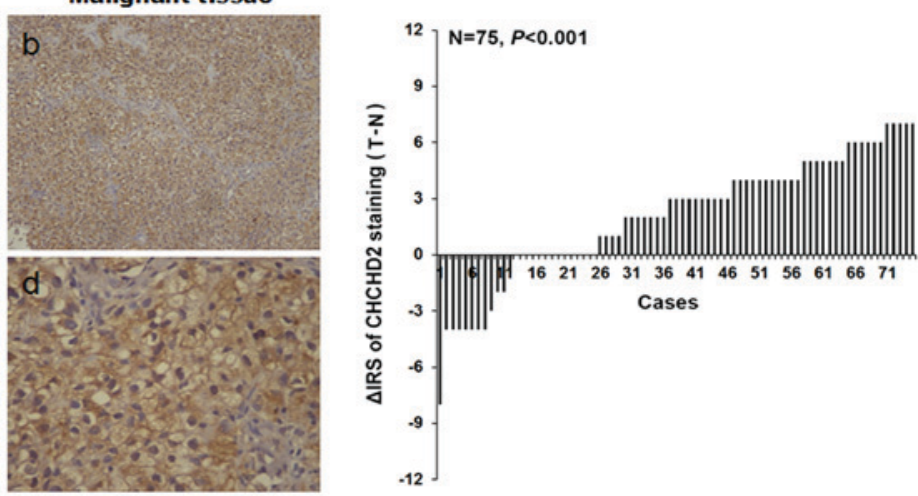

D

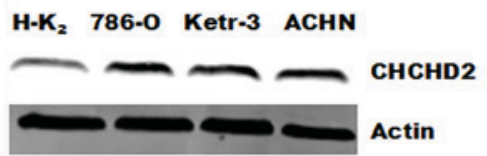

Figure 1. CHCHD2 protein expression was determined in RCC tissues and cells by immunohistochemical staining and western blot analysis. (A) Representative images revealed CHCHD2 immunohistochemical staining in (b and d) RCC maligant tissues and (a and c) tumor adjacent tissues, which were taken at different magnifications (Top panel, x100; bottom panel, x400). (B) The distribution of the difference in CHCHD2 staining $\left(\Delta \mathrm{IRS}=\mathrm{IRS}_{\mathrm{T}}-\mathrm{IRS}_{\mathrm{N}}\right.$ ). Immunoreactivity score (IRS) of CHCHD2 staining was available from 75 pairs of tissues. P-values were calculated with the $\chi^{2}$ test. (C) Whole-cell protein extracts were further prepared from three paired tumor adjacent normal renal tissues and RCC tissues. The CHCHD2 protein level was determined by western blot analysis. (D) In contrast to normal renal HK-2 cell, the CHCHD2 expression is increased in RCC cell lines. RCC, renal cell carcinoma; CHCHD2, coiled-coil-helix-coiled-co il-helix domain-containing protein 2; N, normal renal tissues; T, RCC tissues; IRS, immunoreactivity score.

$48 \mathrm{~h}$ after transfection. The VEGF concentration was measured using a Quantikine ELISA kit (catalog no. DVE00; R\&D Systems, Inc., Minneapolis, MN, USA) according to the manufacturer's protocol.

Statistical analysis. All the data in the figures and text are presented as the mean \pm standard deviation from $>3$ independent experiments. The differences in the TMA were analyzed using SPSS software (version 16.0; SPSS, Inc., Chicago, IL, USA). The association between staining of CHCHD2, and the clinicopathological parameters of the patients with suprarenal epithelioma, including age, sex, World Health Organization grade and histological type $(10,11)$, was evaluated by the $\chi^{2}$ test. Differences in treatment groups were assessed by two-way analysis of variance followed by the Dunnett's test. $\mathrm{P}<0.05$ was considered to indicate a statistically significant difference.

\section{Results}

CHCHD2 expression is increased in human RCC tissues and cell lines. The expression levels of $\mathrm{CHCHD} 2$ were evaluated in human RCC. Immunohistochemical staining was performed in RCC tissues and paired non-cancerous tissues. The representative images presented in Fig. 1A revealed that CHCHD2 protein in cytoplasm was stained brown. Significantly higher expression of CHCHD2 was observed in the carcinoma tissues $(\mathrm{P}<0.001$; Fig. 1B). In order to confirm the aforementioned observation, western blotting was performed using three RCC tissues together with paired non-cancerous tissues. Notably, the level of CHCHD2 protein was markedly increased in malignant tumor tissues compared with non-cancerous tissues (Fig. 1C). Additionally, the differences in expression of CHCHD2 between normal renal cells and carcinoma cells were investigated. Western blotting was

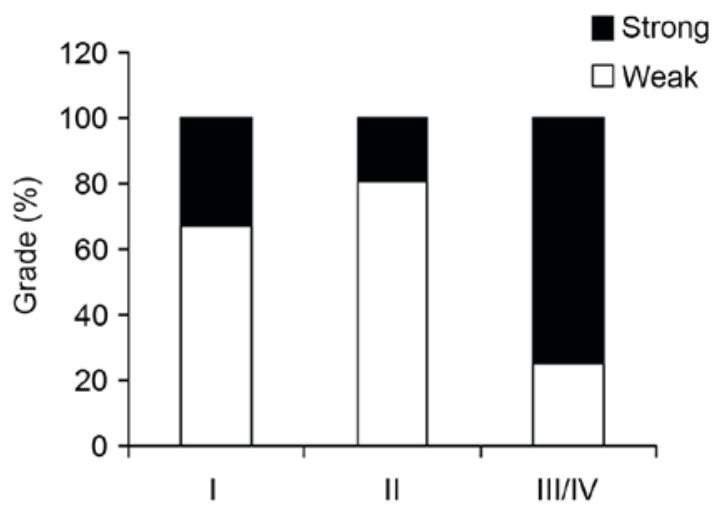

Figure 2. Association between CHCHD2 expression and clinicopathologic parameters in RCC. The immunohistochemical analysis identified that increased CHCHD2 expression was markedly associated with tumor grade $\left(\mathrm{P}<0.001, \chi^{2}\right.$ test, comparing I, II vs. III-IV). CHCHD2, coiled-coil-helix-coi led-coil-helix domain-containing protein 2.

performed to measure the CHCHD2 expression in distinct renal cell lines. The results identified that $\mathrm{CHCHD} 2$ expression levels were markedly increased in human 786-O, Ketr-3 and ACHN RCC cells, compared with HK-2 normal renal cells (Fig. 1D). This result was consistent with the level of $\mathrm{CHCHD} 2$ protein expression in RCC tissues.

Association between CHCHD2 expression levels and clinicopathological parameters. To further investigate the possible function of CHCHD2 in RCC, the associations between CHCHD2 expression in primary tumor tissue samples from 75 patients and clinicopathological parameters were assessed. The mean age of the patients with suprarenal epithelioma was 58.7 years (50 males and 25 females with a median age of 
A

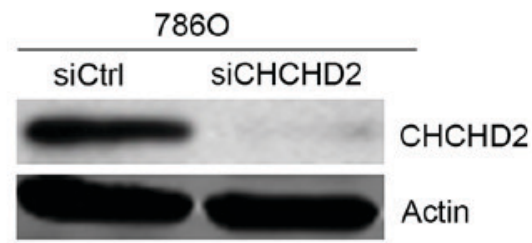

C
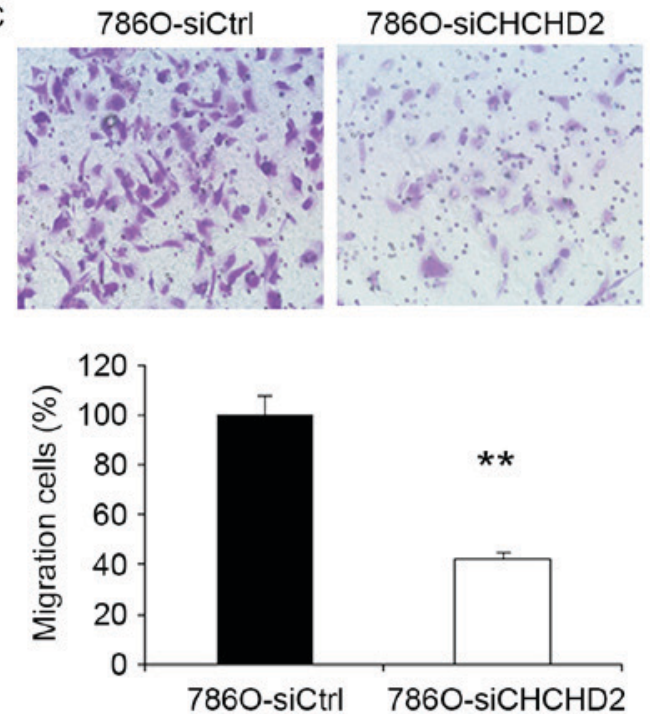

E
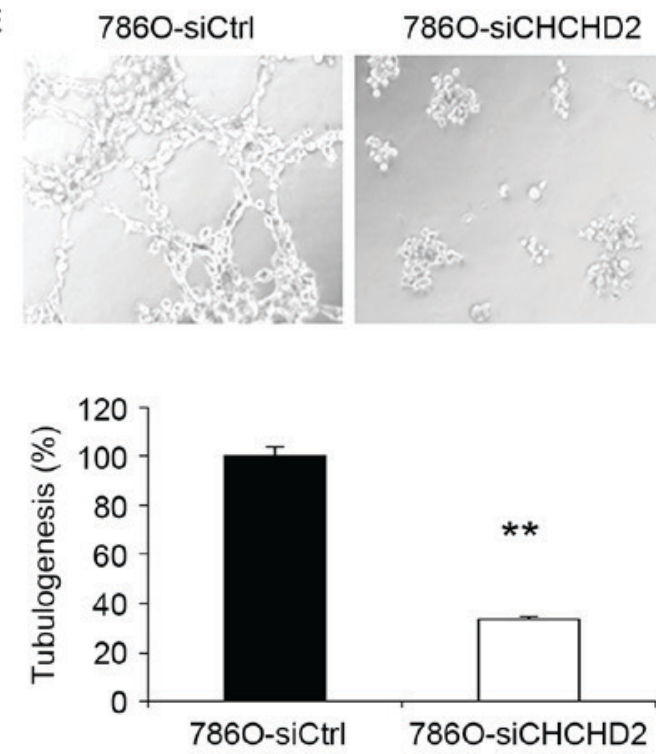

B

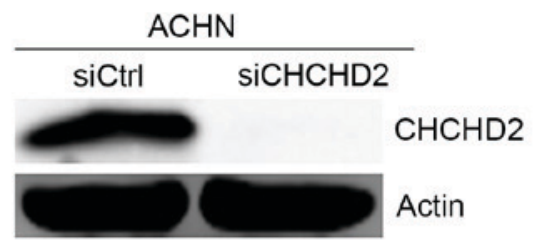

D
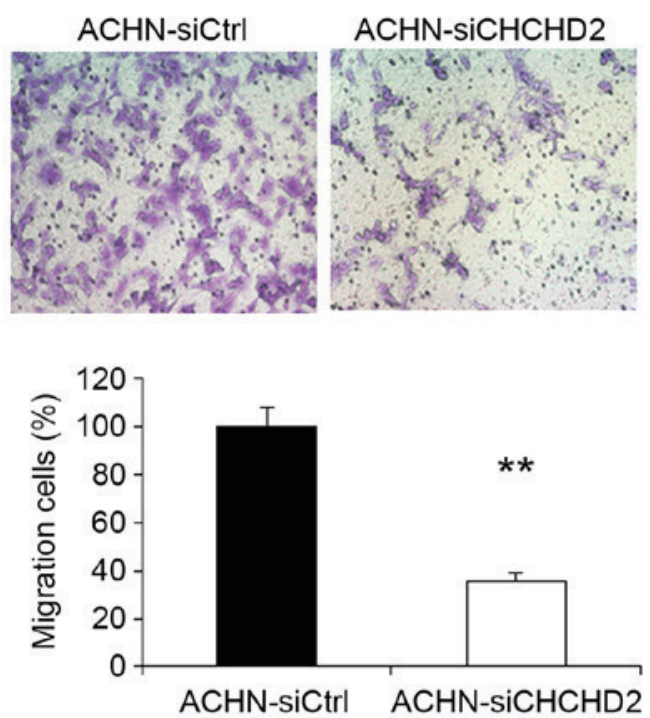

F

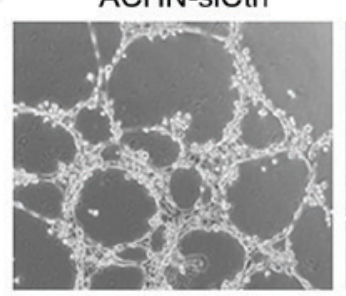

ACHN-SiCHCHD2
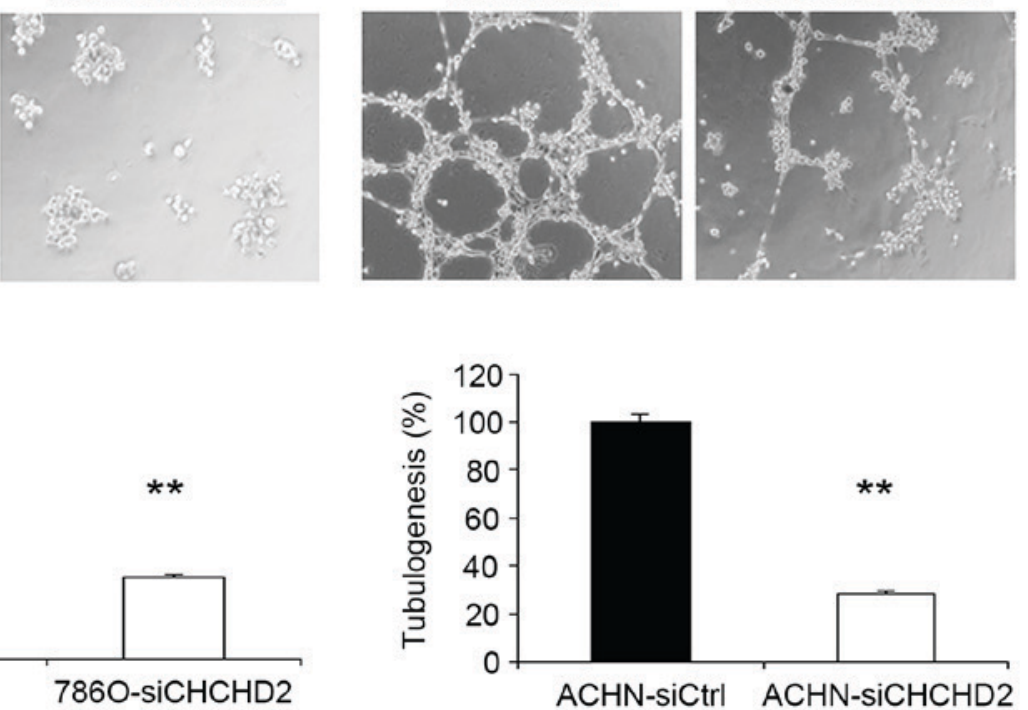

Figure 3. Knockdown of CHCHD2 inhibits the motility and angiogenesis of the 786-O and ACHN cells. (A) Western blot analysis of the relative protein level of CHCHD2 in CHCHD2-knockdown (siCHCHD2) and control siRNA (siCtrl) groups for the (A) 786-O and (B) ACHN cell lines. (C and D) A cell migration assay revealed that knockdown of $\mathrm{CHCHD} 2$ decreased the ability of cells to migrate through a Boyden chamber in 786-O and ACHN cell. (E and F) Knockdown of $\mathrm{CHCHD} 2$ decreased angiogenesis in 786-O and ACHN cells. All experiments were performed in triplicate. Data are presented as mean \pm standard error of the mean. ${ }^{* *} \mathrm{P}<0.01$ for siCHCHD2 vs. siCtrl. CHCHD2, coiled-coil-helix-coiled-coil-helix domain-containing protein 2.

56 years; range, 29-82 years). The comparisons of numerous clinicopathological characteristics with CHCHD2 protein expression are presented in Table I. The results revealed that CHCHD2 staining was significantly increased in tumor grade II-IV compared with tumor grade I ( $\mathrm{P}<0.001$; Fig. 2). Although TNM stage is an important prognostic marker for patients with RCC, no significant association was observed between $\mathrm{CHCHD} 2$ expression and age $(\mathrm{P}=0.725)$, sex $(\mathrm{P}=0.870)$, tumor size $(\mathrm{P}=0.301)$ and TNM stage $(\mathrm{P}=0.166)$.
Knockdown of CHCHD2 inhibits migration of RCC cells in vitro. To further investigate the function of $\mathrm{CHCHD} 2$ in tumor cell migration, human 786-O and ACHN cells were transfected with control siRNA and CHCHD2 siRNA. At $48 \mathrm{~h}$ after transfection, $\mathrm{CHCHD} 2$ protein was markedly downregulated in cancer cells (Fig. 3A and B). As presented in Fig. 3C, silencing of CHCHD2 significantly inhibited the 786-O cells ability to migrate through the Boyden chambers. In order to examine the effect of CHCHD2 knockdown on other RCC 
A

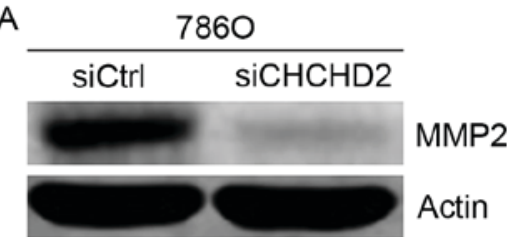

C

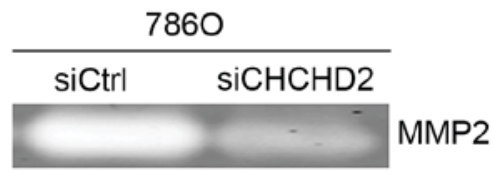

E

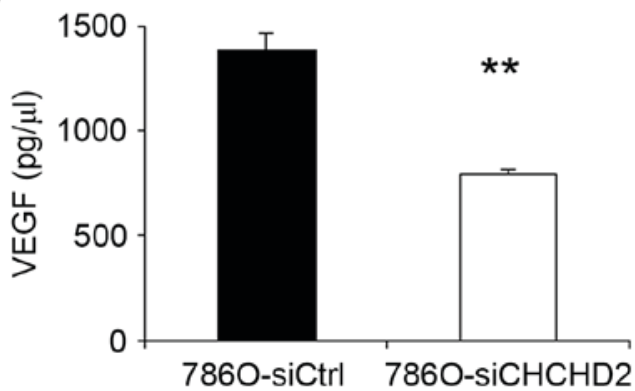

B

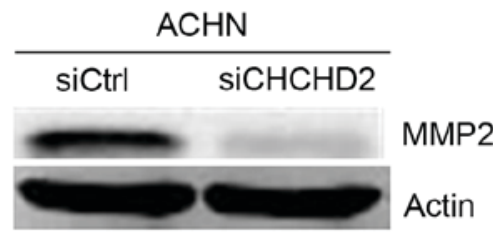

D

$\frac{\mathrm{ACHN}}{\text { siCtrl siCHCHD2 }} \begin{aligned} & \text { MMP2 } \\ & \end{aligned}$

$\mathrm{F}$

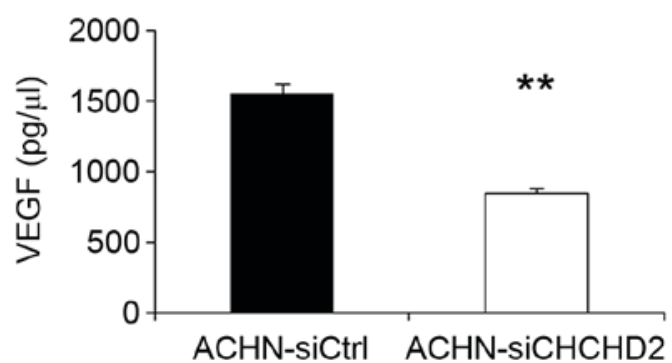

Figure 4. Knockdown of CHCHD2 decreases the expression and ability of MMP-2 protein and suppresses VEGF secretion in 786O and ACHN cells. (A and B) Western blot analysis identified that CHCHD2 knockdown decreased the expression level of MMP-2. (C and D) Gelatin zymography confirmed that transfecting with CHCHD2-siRNA may result in decreasing the MMP-2 protein ability when compared to the control. (E and F) In siCHCHD2 and control group for RCC cell lines, the VEGF secretion was markedly decreased in 786-O-siCHCHD2 cells and ACHN-siCHCHD2 cells, respectively. All experiments were carried out in triplicate. ${ }^{* *} \mathrm{P}<0.01$ for siCHCHD2 vs. siCtrl. CHCHD2, coiled-coil-helix-coiled-coil-helix domain-containing protein 2; VEGF, vascular endothelial growth factor.

cells, ACHN cell migratory assays were performed. The data gathered revealed that the migratory ability of ACHN cells was also suppressed by silencing of CHCHD2 (Fig. 3D).

Knockdown of CHCHD2 suppresses angiogenesis in RCC cells. Angiogenesis induces the growth and metastasis of tumors (14). Therefore, the effect of angiogenesis following knockdown of CHCHD2 was investigated. The endothelial cell tube formation assay (Fig. 3E and F) identified that conditioned medium from CHCHD2 knockdown cancer cells significantly reduced the average number of complete tubular structures formed by HUVECs compared with those of control cells.

Knockdown of CHCHD2 decreases MMP-2 expression, activities and VEGF secretion in RCC cells. In order to improve understanding of the mechanism of siCHCHD2-induced cell migration and angiogenesis inhibition in RCC cells, the MMP expression levels and activities, and VEGF expression levels in 786-O and ACHN cells were measured. The invasive ability of cancer cells may be regulated by MMPs (15-17), and VEGF is considered one of the most prominent pro-angiogenic factors (18-21). The results of the present study revealed that MMP-2 levels were markedly suppressed in 786-O-siCHCHD2 and ACHN-siCHCHD2 cells compared with the control cells (Fig. 4A and B). The activities of MMP-2 protein were also markedly decreased in 786-O and ACHN cells transfected with siCHCHD2 using gelatin zymography (Fig. 4C and D). Additionally, it was identified that VEGF secretion was significantly suppressed in 786-O and ACHN cells transfected with siCHCHD2 compared with the control cells (Fig. 4E and F).

\section{Discussion}

Previous research identified that Cytochrome c oxidase (COX) is the terminal enzyme of the electron transport chain, and COX subunit 4 (COX4) is a key regulatory subunit (22). Aras et al (22) demonstrated that oxygen responsive element (ORE) is a highly conserved 13-bp sequence in the proximal promoter of the tissue-specific (predominantly lung) COX4 isoform 2, and further confirmed that $\mathrm{CHCHD} 2$, as a transcription factor, bound this conserved ORE and activated the ORE at $4 \%$ oxygen. Seo et al (8) reported that CHCHD2 protein was able to mutually regulate the balance of cell migration by directly interacting with hyaluronic acid-binding protein 1, and CHCHD2 was identified as a novel cell migration determinant using an in vitro functional genetic selection strategy. In addition, CHCHD2 is involved in mitochondrial function (23) and has been identified in a screen for HTT interacting proteins $(24,25)$. Liu et al (9) demonstrated that $\mathrm{CHCHD} 2$ was able to inhibit apoptosis by interacting with $\mathrm{Bcl}-\mathrm{xL}$ to regulate BAX activation. Furthermore, increasing evidence suggests a potential function of $\mathrm{CHCHD} 2$ in CHCHD2-Bcl-xL protein interactions.

In the present study, CHCHD2 expression was first examined at the protein level in RCC cells and tissues using western blotting. The results indicate that the expression of CHCHD2 protein was more abundant in suprarenal epithelioma cells and tissues compared with that of the normal renal cells and tissues. The expression level of CHCHD2 was markedly associated with tumor grade in data gathered from a TMA. However, little is known about the association between CHCHD2 expression and patients with RCC, and the functional role 
of $\mathrm{CHCHD} 2$ in the progress of $\mathrm{RCC}$ remains unresolved. To determine whether $\mathrm{CHCHD} 2$ regulates the development of RCC, RNA interference (RNAi) was used in further functional studies $(26,27)$. CHCHD2 siRNA or control siRNA were transfected into 786-O and ACHN cells to observe the effect on the cells ability to migrate. The results demonstrate that silencing CHCHD2 with RNAi resulted in significantly reduced cell migration rates in both cell lines. To investigate the associated mechanism of this phenomenon in 7860 and ACHN cells, the level of various movement-associated proteins was analyzed. MMPs may allow cancer cell to migrate and invade by virtue of degrading components of the basement membrane and the extracellular matrix $(28,29)$. Therefore, it was hypothesized that siCHCHD2-induced inhibition of cell migration was also associated with MMPs. The results suggested an association between CHCHD2 and migration of RCC. In gelatin zymography, the activities of MMP-2 protein were markedly decreased in 7860-siCHCHD2 and ACHN-siCHCHD2 cells compared with the control groups.

Previous research reported that $30 \%$ of RCC patients develop metastatic disease following surgical resection (4), and the growth and metastasis of neoplasms is dependent on angiogenesis $(30,31)$. The results of the present study identified that knockdown of CHCHD2 suppressed angiogenesis, and further research revealed that knockdown of CHCHD2 inhibited VEGF secretion. VEGF is the most prominent of these pro-angiogenic factors (19-21). Additionally, several studies reported that increased VEGF expression was identified to be associated with poorer prognosis in RCC (32). Therefore, these findings suggest an association between CHCHD2, and migration and angiogenesis of RCC.

In summary, an increase of CHCHD2 expression was observed in the groups presenting with RCC as compared with the normal renal cells and tissues. This observation implied a potential function of CHCHD2 in the development and progression of renal carcinoma. The gathered data demonstrated that migration of RCC cells was promoted through increasing MMP-2 protein expression and ability. The knockdown of CHCHD2 suppressed angiogenesis of RCC cells through decreasing VEGF secretion. Although the underlying molecular mechanisms of $\mathrm{CHCHD} 2$ promoting suprarenal epithelioma cell migration and angiogenesis require further investigation, CHCHD2 may be eventually be utilized as a novel molecular marker and a potential target in the therapy for RCC.

\section{Acknowledgements}

Not applicable.

\section{Funding}

The present study was supported by a grant from the National Natural Science Foundation of China (no. 81201637).

\section{Availability of data and materials}

The datasets used and/or analyzed during the current study are available from the corresponding author on reasonable request.

\section{Authors' contributions}

LZ designed the study and applied for approval from the Research Ethics Board. ZL recruited the patients and collected the data. QC and DQ analyzed the data and prepared draft figures, tables and the manuscript draft.

\section{Ethics approval and consent to participate}

The Institutional Review Board of Xuzhou Medical University approved the present study, conducted in accordance with the approved guidelines. All patients provided written informed consent for their colorectal tissue samples to be used in the present study.

\section{Patient consent for publication}

All patients provided written informed consent for their colorectal tissue samples to be used in the present study.

\section{Competing interests}

The authors declare that they have no competing interests.

\section{References}

1. Chow WH, Dong LM and Devesa SS: Epidemiology and risk factors for kidney cancer. Nat Rev Urol 7: 245-257, 2010.

2. Volpe A and Patard JJ: Prognostic factors in renal cell carcinoma. World J Urol 28: 319-327, 2010.

3. Rathmell WK and Godley PA: Recent updates in renal cell carcinoma. Curr Opin Oncol 22: 250-256, 2010.

4. Al-Ali BM, Ress AL, Gerger A and Pichler M: MicroRNAs in renal cell carcinoma: Implications for pathogenesis, diagnosis, prognosis and therapy. Anticancer Res 32: 3727-3732, 2012.

5. Zisman A, Pantuck AJ, Wieder J, Chao DH, Dorey F, Said JW, deKernion JB, Figlin RA and Belldegrun AS: Risk group assessment and clinical outcome algorithm to predict the natural history of patients with surgically resected renal cell carcinoma. J Clin Oncol 20: 4559-4566, 2002.

6. Rini BI, Campbell SC and Escudier B: Renal cell carcinoma. Lancet 373: 1119-1132, 2009.

7. Baughman JM, Nilsson R, Gohil VM, Arlow DH, Gauhar Z and Mootha VK: A computational screen for regulators of oxidative phosphorylation implicates SLIRP in mitochondrial RNA homeostasis. PLoS Genet 5: e1000590, 2009.

8. Seo M, Lee WH and Suk K: Identification of novel cell migration-promoting genes by a functional genetic screen. FASEB J 24: 464-478, 2010.

9. Liu Y, Clegg HV, Leslie PL, Di J, Tollini LA, He Y, Kim TH, Jin A, Graves LM, Zheng J and Zhang Y: CHCHD2 inhibits apoptosis by interacting with $\mathrm{Bcl}-\mathrm{x} \mathrm{L}$ to regulate $\mathrm{Bax}$ activation. Cell Death Differ 22: 1035-1046, 2015.

10. Guinan P, Sobin LH, Algaba F, Badellino F, Kameyama S, MacLennan G and Novick A: TNM staging of renal cell carcinoma: Workgroup No 3. Union International Contre le Cancer (UICC) and the American Joint Committee on Cancer (AJCC). Cancer 80: 992-993, 1997.

11. Sobin LH and Fleming ID: TNM classification of malignant tumors, fifth edition (1997) union internationale contre le cancer and the American joint committee on cancer. Cancer 80: 1803-1804, 1997.

12. Baumann P,Cremers N, Kroese F, Orend G, Chiquet-Ehrismann R, Uede T, Yagita H and Sleeman JP: CD24 expression causes the acquisition of multiple cellular properties associated with tumor growth and metastasis. Cancer Res 65: 10783-10793, 2005.

13. Chu YW, Yang PC, Yang SC, Shyu YC, Hendrix MJ, Wu R and Wu CW: Selection of invasive and metastatic subpopulations from a human lung adenocarcinoma cell line. Am J Respir Cell Mol Biol 17: 353-360, 1997. 
14. Wachsberger P, Burd R and Dicker AP: Tumor response to ionizing radiation combined with antiangiogenesis or vascular targeting agents: Exploring mechanisms of interaction. Clin Cancer Res 9: 1957-1971, 2003.

15. Bai J, Mei PJ, Liu H, Li C, Li W, Wu YP, Yu ZQ and Zheng JN: BRG1 expression is increased in human glioma and controls glioma cell proliferation, migration and invasion in vitro. J Cancer Res Clin Oncol 138: 991-998, 2012.

16. Kargiotis O, Chetty C, Gondi CS, Tsung AJ, Dinh DH, Gujrati M, Lakka SS, Kyritsis AP and Rao JS: Adenovirus-mediated transfer of siRNA against MMP-2 mRNA results in impaired invasion and tumor-induced angiogenesis, induces apoptosis in vitro and inhibits tumor growth in vivo in glioblastoma. Oncogene 27 4830-4840, 2008

17. Badiga AV, Chetty C, Kesanakurti D, Are D, Gujrati M, Klopfenstein JD, Dinh DH and Rao JS: MMP-2 siRNA inhibits radiation-enhanced invasiveness in glioma cells. PLoS One 6 e20614, 2011.

18. Bergers $\mathrm{G}$ and Benjamin LE: Tumorigenesis and the angiogenic switch. Nat Rev Cancer 3: 401-410, 2003.

19. Dvorak HF: Vascular permeability factor/vascular endothelial growth factor: A critical cytokine in tumor angiogenesis and a potential target for diagnosis and therapy. J Clin Oncol 20 : 4368-4380, 2002.

20. Ferrara N: Vascular endothelial growth factor: Basic science and clinical progress. Endocr Rev 25: 581-611, 2004.

21. Lucio-Eterovic AK, Piao Y and de Groot JF: Mediators of glioblastoma resistance and invasion during antivascular endothelial growth factor therapy. Clin Cancer Res 15: 4589-4599, 2009.

22. Aras S, Pak O, Sommer N, Finley R Jr, Hüttemann M, Weissmann $\mathrm{N}$ and Grossman LI: Oxygen-dependent expression of cytochrome c oxidase subunit 4-2 gene expression is mediated by transcription factors RBPJ, CXXC5 and CHCHD2. Nucleic Acids Res 41: 2255-2266, 2013.
23. Feyeux M, Bourgois-Rocha F, Redfern A, Giles P, Lefort N, Aubert S, Bonnefond C, Bugi A, Ruiz M, Deglon N, et al: Early transcriptional changes linked to naturally occurring Huntington's disease mutations in neural derivatives of human embryonic stem cells. Hum Mol Genet 21: 3883-3895, 2012.

24. Aras S, Bai M,Lee I, Springett R, Hüttemann M and Grossman LI: MNRR1 (formerly CHCHD2) is a bi-organellar regulator of mitochondrial metabolism. Mitochondrion: 43-51, 2015.

25. Kaltenbach LS, Romero E, Becklin RR, Chettier R, Bell R, Phansalkar A, Strand A, Torcassi C, Savage J, Hurlburt A, et al: Huntingtin interacting proteins are genetic modifiers of neurodegeneration. PLoS Genet 3: e82, 2007.

26. Hannon GJ: RNA interference. Nature 418: 244-251, 2002.

27. Elbashir SM, Harborth J, Weber K and Tuschl T: Analysis of gene function in somatic mammalian cells using small interfering RNAs. Methods 26: 199-213, 2002.

28. Rundhaug JE: Matrix metalloproteinases and angiogenesis. J Cell Mol Med 9: 267-285, 2005.

29. Deryugina EI and Quigley JP: Matrix metalloproteinases and tumor metastasis. Cancer Metastasis Rev 25: 9-34, 2006.

30. Folkman $\mathrm{J}$ : What is the evidence that tumors are angiogenesis dependent? J Natl Cancer Inst 82: 4-6, 1990.

31. Fidler IJ: Angiogenesis and cancer metastasis. Cancer J6 (Suppl2): S134-S141, 2000.

32. Jacobsen J, Rasmuson T, Grankvist K and Ljungberg B: Vascular endothelial growth factor as prognostic factor in renal cell carcinoma. J Urol 163: 343-347, 2000.

This work is licensed under a Creative Commons Attribution-NonCommercial-NoDerivatives 4.0 International (CC BY-NC-ND 4.0) License. 